\title{
Riesgos laborales en personal de limpieza pública durante el Covid-19
}

\author{
Gloria Isabel Monzón Alvarez \\ ORCID: https://orcid.org/0000-0001-8712-5188 \\ gmonzon@unsa.edu.pe \\ Universidad Nacional de San Agustín de Arequipa \\ Arequipa, Perú \\ Ana Rosario Miaury Vilca \\ ORCID: https://orcid.org/0000-0002-2992-1239 \\ amiaury@unsa.edu.pe \\ Universidad Nacional de San Agustín de Arequipa \\ Arequipa, Perú
}

\author{
Miguel Ángel Pacheco Quico \\ ORCID: https://orcid.org/0000-0001-9317-8518 \\ mpachecoq@unsa.edu.pe \\ Universidad Nacional de San Agustín de Arequipa \\ Arequipa, Perú \\ Hilda Lizbeth Pinto Pomareda, \\ ORCID: https://orcid.org/0000-0002-1719-4863 \\ hpintop@unsa.edu.pe \\ Universidad Nacional de San Agustín de Arequipa \\ Arequipa, Perú
}

\author{
Santiago Felipe Torres Aza \\ ORCID: https://orcid.org/0000-0002-6984-1356 \\ Santiago.torresa@ucsm.edu.pe \\ Universidad Católica Santa María \\ Arequipa, Perú
}

Recibido (02/02/21), Aceptado (19/02/21)

\begin{abstract}
Resumen: Se presentan los resultados de un estudio basado en el análisis de los riesgos laborales a los que están expuestos el personal de limpieza pública durante los eventos del Covid-19, tomando en cuenta las características socio laborales. En el estudio participaron 212 colaboradores de dos municipios de la ciudad de Arequipa, se les aplicó la escala RL-14 y se encontró que existe alta exposición a riesgos laborales, observándose además que hay varios trabajadores afectados por otras enfermedades ocupacionales y contagiados de Covid-19. Se concluye que, el personal de limpieza pública en Arequipa presenta riesgos laborales por el tipo de trabajo que desarrollan en las vías públicas, siendo los principales riesgos los psicosociales, físicos, ergonómicos y condiciones inseguras.
\end{abstract}

Palabras Clave: Riesgos laborales, trabajadores públicos, Covid-19.

\section{Occupational risks in public cleaning personnel during Covid-19}

\begin{abstract}
The results of a study based on the analysis of the occupational risks to which public cleaning personnel are exposed during the Covid-19 events are presented, taking into account the socio-labor characteristics. 212 employees from two municipalities in the city of Arequipa participated in the study, the RL-14 scale was applied to them and it was found that there is high exposure to occupational risks, also observing that there are several workers affected by other occupational diseases and infected with Covid- 19 . It is concluded that public cleaning personnel in Arequipa present occupational risks due to the type of work they carry out on public roads, the main risks being psychosocial, physical, ergonomic and unsafeconditions.
\end{abstract}

Keywords: Occupational risks, public workers, Covid-19. 


\section{I.INTRODUCCIÓN}

El impacto del Covid-19 en el mundo, ha afectado el normal desenvolvimiento de las personas en los diferentes espacios de interacción social, observándose que no sólo perturba la salud sino que también afecta las condiciones en el trabajo [1], en muchos casos los colaboradores perdieron sus trabajos, en otros casos por no perder el trabajo se adecuaron a las nuevas disposiciones de sus empleadores, algunos realizaron actividades desde sus hogares y otros tuvieron que salir a trabajar a las calles en contra de su voluntad, además, que los sueldos y salarios se redujeron a menos de la mitad.

Es evidente que los trabajadores en general sienten la preocupación ante la posibilidad de contagiarse de Covid-19, por ello, ante la incertidumbre de perder el empleo tienen la necesidad de trabajar fuera del hogar tomando las medidas necesarias para poder solventar las principales necesidades básicas de sus familias [2], no obstante, la probabilidad de contagio sigue siendo alta.

El personal de limpieza de los municipios urbanos está integrado por los barrenderos, los que recolectan y reciclan los residuos sólidos, los que fumigan los ambientes públicos, los que realizan mantenimiento de parques y jardines entre otros. El tipo de actividad incrementa las amenzas de contagio por el Covid-19. Ssegún el último reporte de la organización CONVOCA más del $40 \%$ de trabajadores contrajo el virus en su puesto de trabajo [3].

A pesar de que el Ministerio de Trabajo y Promoción del Empleo en el Perú [4] emitió el reglamento de seguridad y salud en el trabajo de los obreros municipales (D.S. 017-2017-TR), estos esfuerzos no se ven reflejados en las condiciones laborales del personal de limpieza pública, percibiéndose que los niveles de protección contra los riesgos para su seguridad y salud siguen siendo bajos, debido a la ausencia de implementos de protección, jornadas laborales largas, escaso reconocimiento de su labor y sobre todo el estereotipo social del que son objeto.

Así también, la mayoría de los colaboradores de limpieza desarrollan sus actividades laborales en las vías públicas, incrementando el nivel de exposición a factores de riesgo tanto ambientales, psicológicos, físicos y sociales, que pueden afectar su salud, adquiriendo además otras enfermedades ocupacionales, como las músculo esqueléticas, gastrointestinales y respiratorias [5], así como, contagiarse por el virus debido al contacto directo con los desechos.

En tal sentido, de acuerdo con el contexto y la situación actual de esta población el siguiente trabajo muestra los resultados de un estudio cuyo objetivo consistió en analizar los riesgos laborales a los que están expuestos el personal de limpieza pública, tomando en cuenta las características socio laborales durante la pandemia del Covid-19. En la investigación se utilizó el diseño metodológico cuantitativo, descriptivo - comparativo, transversal, no experimental [6]. La información se recolectó durante los meses de agosto a septiembre del año 2020.

El trabajo consta de cuatro secciones; en la primera se han descrito los elementos básicos que componen el estudio, en la segunda se exponen los aspectos teóricos, en la tercera sección se describen los aspectos metodológicos y finalmente se exponen los resultados y conclusiones.

\section{II.DESARROLLO}

Los riesgos laborales son situaciones que existen en los ambientes de trabajo y que presentan alta probabilidad de dañar la salud de las personas [7], no se distingue profesión ni ocupación ya que cualquier trabajador puede estar sometido al riesgo de enfermarse o sufrir un accidente con la labor que desempeña, siendo la salud ocupacional la que se encarga de estudiarlo [8].

Las nuevas condiciones laborales propuestas por las organizaciones han generado nuevos riesgos que son causados por factores como contratos precarios, inseguridad laboral, desmotivación, la intensificación horaria, las deficientes relaciones laborales y sobre todo la escasa valoración del trabajo que realiza el colaborador [7].

En relación con los principales riesgos laborales, los riesgos psicosociales son de mucha relevancia para el análisis dado que las situaciones adversas a los que están expuestos los colaboradores por la presión laboral puede generar niveles elevados de estrés o patologías psicológicas (ansiedad, depresión, burnout, etc.) que ponen en riesgo la salud mental del personal [9]. La figura 1 muestra los principales riesgos laborales de carácter psicosocial, se puede evidenciar que los más destacados incluyen el tiempo de la jornada laboral, las condiciones laborales y los factores externos que intervienen con el entorno de trabajo. Así pues, los empleados del sector de limpieza presentan altos riesgos por las desmejoras en las condiciones laborales, las largas jornadas y la situación inevitable de la pandemia, que incrementa todo tipo de situación estresante en los trabajadores. 


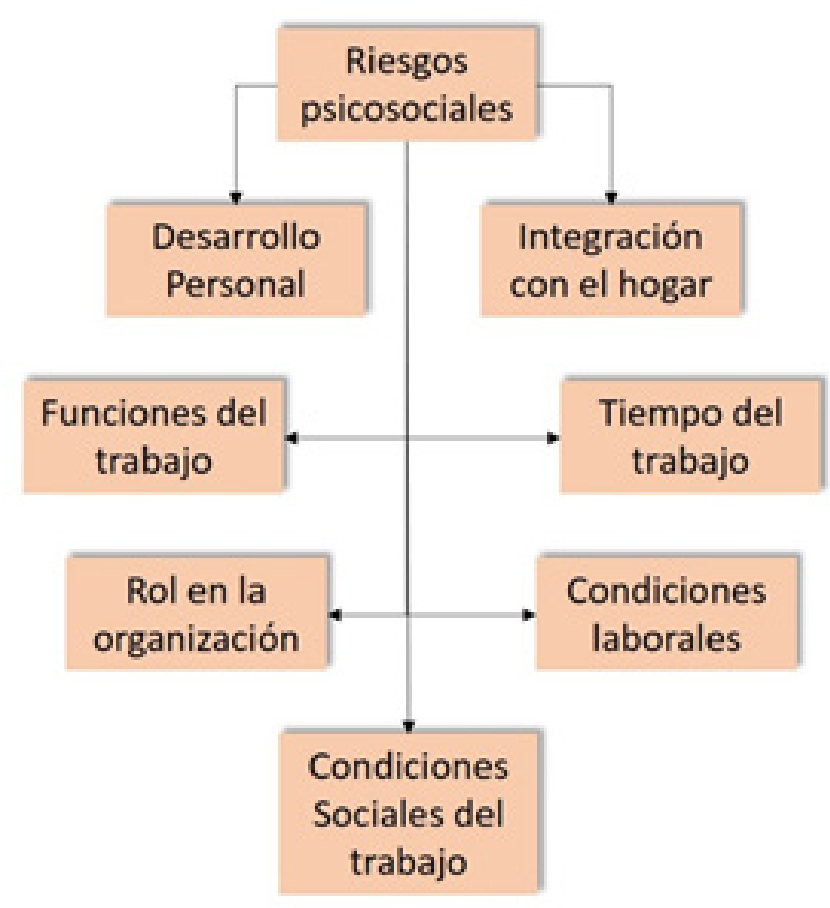

Figura 1. Principales riesgos laborales.

Así también, los riesgos físicos ambientales se producen por el nivel de exposición a la iluminación, el ruido, las radiaciones y las temperaturas extremas a los que están sometidos los colaboradores sobre todo de limpieza en las calles. También están presentes los riesgos ergonómicos, los que se producen por los movimientos, las cargas físicas y los esfuerzos que realizan los colaboradores de acuerdo a su puesto de trabajo, trayendo consecuencias como los trastornos músculo esqueléticos, produciendo daños en articulaciones, tendones, músculos, nervios, dolores intensos en diferentes partes del cuerpo [10], entre otras dolencias asociadas.

Además, se deben considerar algunos otros riesgos de exposición como son los actos inseguros, que es cuando el trabajador no usa el equipo de protección personal y la condición insegura, que es cuando la empresa u organización no proporciona el equipo de protección personal, lo que puede ocasionar hasta la muerte [11].

En este trabajo se evaluaron los aspectos relacionados con la información y la formación de los trabajadores, referente a toda la capacitación relacionada con los riesgos laborales a los que pudiese estar expuesto el trabajador. También se evaluaron las patologías psicofisiológicas, que se refieren a las enfermedades profesionales como el músculo esquelético, sistema nervioso central, respiratorias y las psicológicas [12].

Con respecto a las variables sociodemográficas y los riesgos laborales producidos en el trabajo no se han encontrado específicamente estudios en personal de limpieza que demuestren si existe mayor riesgo de acuerdo a la edad, el género, la ocupación o la condición laboral, sin embargo, el estudio propuesto es de interés para diferentes disciplinas que se encargan de estudiar el comportamiento humano y organizacional a fin de profundizar y explicar los principales riesgos a los que se exponen los colaboradores y en qué situación se encuentran.

\section{III.METODOLOGÍA}

En este trabajo participaron 212 colaboradores de limpieza pública de dos municipalidades distritales de la ciudad de Arequipa. La edad promedio de los colaboradores fue de 33 años con una desviación de \pm 9.06 dentro de un rango de 19 a 61 años. Con respecto al sexo de los colaboradores el $43.9 \%$ fueron mujeres y el $56.1 \%$ varones. Así también, el $41 \%$ de los colaboradores presentaron la condición laboral de nombrados y $59 \%$ con contrato CAS (contrato administrativo de servicios).

Para medir la situación a exposición de riesgos laborales, se utilizó la escala RL-14 [12], el instrumento consta de 14 ítems de respuesta tipo Likert, las puntuaciones de los ítems varían de acuerdo a la situación ( siempre $=5$, muchas veces $=4$, algunas veces $=3$, solo alguna vez $=2$, nunca $=1$ ), la escala se distribuye en 2 dimensiones: dimensión información y formación y dimensión patologías psicofisiológicas, siendo las puntuaciones entre 14 (no expuesto) a 70 (afectado). Así mismo, en el instrumento se incluyeron características socio laborales como edad, sexo, condición laboral y puesto de trabajo, además se consignaron los datos del consentimiento informado.

Para la versión adaptada a la muestra local, se obtuvieron los niveles de confiabilidad a través del coeficiente alfa de Cronbach, obteniendo un valor de $\alpha=$ 0.902 (excelente) [13].

El estudio cumplió con todos los rigores de autorizaciones necesarias, tanto del Municipio como de los trabajadores. Asegurando una conducta ética y responsable.

\section{RESULTADOS}

Para el análisis estadístico se utilizó el programa JASP 0.13.1.0. La exploración de la normalidad de los datos a través de la prueba de Kolmogorov-Smirnov [14] demostró que no existe distribución normal, por lo tanto, se utilizaron estadísticos no paramétricos. Se realizó el análisis categórico de la situación de exposición a riesgos laborales y sus dimensiones, en relación con el análisis comparativo, para encontrar diferencias sig- 
nificativas según características socio laborales, se utilizaron los estadígrafos U de Mann-Whitney (para dos muestras independientes) [15], la $\mathrm{H}$ de Kruskall Wallis (para más de dos muestras independientes). Además, se estimó el tamaño del efecto para U (probabilidad de superioridad) y para la $\mathrm{H}$ (eta cuadrada) [16].

En la tabla 1 se describe la exposición a riesgos laborales que presenta el personal de limpieza durante el Covid-19, los datos demuestran que, en el riesgo laboral general, los colaboradores se encuentran expuestos en un $48.1 \%$, lo que representa un valor importante para los trabajadores, ya que la probabilidad de contagio es alta, considerando este valor. Por otra parte, el 38.7\% están afectados, no necesariamente con el virus, pero si padecen algún problema de salud, lo que representa un valor significativo, y de alguna forma representa un riesgo para los que no presentan problemas de salud, y solo un porcentaje pequeño, del $13.2 \%$ no se encuentran aún expuestos.

Tabla 1. Exposición a riesgos laborales en personal de limpieza pública

\begin{tabular}{lcccccc}
\hline \multirow{2}{*}{ Dimensiones } & \multicolumn{2}{c}{ Afectado } & \multicolumn{2}{c}{ Expuesto } & \multicolumn{2}{c}{ No Expuesto } \\
& $f(x)$ & $\%$ & $f(x)$ & $\%$ & $f(x)$ & $\%$ \\
\hline Riesgo laboral & 82 & $38.7 \%$ & 102 & $48.1 \%$ & 28 & $13.2 \%$ \\
Información y Formación & 64 & $30.2 \%$ & 116 & $54.7 \%$ & 32 & $15.1 \%$ \\
Patologías psicofisiológicas & 54 & $25.5 \%$ & 130 & $61.3 \%$ & 28 & $13.2 \%$ \\
\hline
\end{tabular}

Nota: $\mathrm{f}(\mathrm{x})=$ frecuencia; $\%=$ porcentaje.

$\mathrm{Al}$ evaluar lo relacionado con información y formación, fue posible observar que los colaboradores se encuentran expuestos en un $54.7 \%$ debido a que no reciben la información sobre riesgos laborales, más aun durante la pandemia. Se pudo observar que los trabajadores no reciben la comunicación efectiva sobre los nuevos procesos ni las nuevas herramientas de trabajo, obteniendo que el $30.2 \%$ se siente afectado por esta situación y el $15.1 \%$ no manifiesta exposición significativa.

En la evaluación de las patologías psicofisiológicas, se pudo evidenciar que el $61.3 \%$ se encuentra expuestos, mientras que el $25.5 \%$ está afectado, debido a que han adquirido enfermedades profesionales sobre todo las relacionadas a la categoría músculo esqueléticas originadas por el tipo de trabajo que desarrollan en las vías públicas, así mismo, las condiciones de salud en el país también han repercutido en la salud mental de estos colaboradores porque presentan temor al contagio del virus de Covid-19 (Tabla 2).

Tabla 2. Comparación de la exposición a riesgos laborales según sexo

\begin{tabular}{lccccc}
\hline \multirow{1}{*}{ Dimensiones } & $\begin{array}{c}\text { Hombres } \\
(n=119)\end{array}$ & $\begin{array}{c}\text { Mujeres } \\
(n=93)\end{array}$ & \multicolumn{2}{c}{ Estadigrafo } & \multirow{2}{*}{$P S_{\text {est }}$} \\
\cline { 2 - 5 } & Rango & Rango & $U$ & $p$ & \\
\hline Riesgo laboral & 99.08 & 115.99 & 4651.00 & 0.046 & 0.22 \\
Información y Formación & 99.63 & 115.30 & 4715.00 & 0.064 & 0.18 \\
Patologías psicofisiológicas & 98.05 & 117.32 & 4527.50 & 0.022 & 0.29 \\
\hline
\end{tabular}

Nota: $\mathrm{n}=$ tamaño muestral; Rango $=$ Rango Promedio; $\mathrm{U}=\mathrm{U}$ de Mann Withney; $\mathrm{p}=\mathrm{p}$ valor; PSest= Probabilidad de Superioridad (tamaño del efecto).

Además se compararon la exposición a riesgos laborales durante el Covid-19 según sexo de los colaboradores encontrándose diferencias estadísticamente significativas, en la escala general, se observa que son las mujeres las que presentan mayor exposición al riesgo que los varones (tamaño del efecto pequeño), así mismo, en la evaluación de patologías psicofisiológicas también se encontraron diferencias significativas, siendo también las mujeres las que se encuentran más expuestas y afectadas a los riesgos laborales, que los varones (tamaño del efecto pequeño). Sin embargo, en la evaluación de información y formación no se hallaron diferencias estadísticas, lo que demuestra que tanto hombres y mujeres presentan el mismo nivel de exposición al riesgo, por la ausencia de comunicación sobre el tema por parte de los supervisores y responsables de 
área (Tabla 2).

Al comparar la exposición a riesgos laborales del personal de limpieza durante el Covid-19, tomando en cuenta la condición de trabajo, se encontraron diferencias estadísticamente significativas en la escala general y en sus dimensiones de estudio. Por lo tanto, los colaboradores bajo la condición de contrato de administración por servicios (CAS) se encuentran más expuestos y afectados que los colaboradores con calidad de nombrados, debido a que sus condiciones laborales difieren desde las facilidades para asistir al trabajo hasta las remuneraciones, siendo el personal contratado quien se encuentra más presionado para cumplir con los objetivos del área, obligándolos a realizar jornadas laborales más largas y complejas, lo que afecta la salud y la exposición más prolongada al contagio del virus (tamaño del efecto pequeño a moderado respectivamente) (Tabla 3 ).

Tabla 3. Comparación de la exposición a riesgos laborales según condición de trabajo

\begin{tabular}{lccccc}
\hline \multirow{1}{*}{ Dimensiones } & $\begin{array}{c}\text { Nombrado } \\
(n=87)\end{array}$ & $\begin{array}{c}\text { CAS } \\
(n=125)\end{array}$ & \multicolumn{2}{c}{ Estadigrafo } & \multirow{2}{*}{$P S_{\text {est }}$} \\
\cline { 2 - 5 } & Rango & Rango & $U$ & $p$ & \\
\hline Riesgo laboral & 93.76 & 115.36 & 4329.50 & 0.011 & 0.46 \\
Información y Formación & 92.32 & 116.37 & 4204.00 & 0.005 & 0.36 \\
Patologías psicofisiológicas & 96.07 & 113.79 & 4530.00 & 0.037 & 0.24 \\
\hline
\end{tabular}

Nota: $\mathrm{n}=$ tamaño muestral; Rango $=$ Rango Promedio; $\mathrm{U}=\mathrm{U}$ de Mann Withney; $\mathrm{p}=\mathrm{p}$ valor; PSest= Probabilidad de Superioridad (tamaño del efecto).

En la tabla 4 se compararon los riesgos laborales durante el Covid-19 según edad y puesto de trabajo (más de dos grupos de comparación), a través de las pruebas post hoc, se encontró que según la edad existen diferencias estadísticamente significativas, se determina que los colaboradores mayores se encuentran más expuestos que los otros colaboradores por ser considerados personal de alto riesgo con la probabilidad de contagiarse (tamaño del efecto moderado).

Tabla 4. Comparación de la exposición a riesgos laborales según edad y puesto de trabajo

\begin{tabular}{|c|c|c|}
\hline & $\begin{array}{l}\text { Grupo de edad } \\
\text { (n) } \\
\text { Rango promedio }\end{array}$ & $\begin{array}{l}\text { Puesto de trabajo } \\
\text { (n) } \\
\text { Rango promedio }\end{array}$ \\
\hline \multirow{4}{*}{ Riesgos laborales } & $\begin{array}{c}\text { Entre } 18 \text { y } 35 \text { años } \\
(n=23) \\
87.88 \\
\end{array}$ & $\begin{array}{c}\text { Parques y jardines } \\
(n=60) \\
127.33 \\
\end{array}$ \\
\hline & $\begin{array}{c}\text { Entre } 35 y 50 \text { años } \\
(n=127) \\
103.70 \\
\end{array}$ & $\begin{array}{c}\text { Servicios generales } \\
\left(\begin{array}{c}n=9) \\
89.69\end{array}\right. \\
\end{array}$ \\
\hline & $\begin{array}{c}\text { Más de } 51 \text { años } \\
\qquad \begin{array}{c}(n=62) \\
172.17\end{array}\end{array}$ & $\begin{array}{c}\text { Limpieza pública } \\
(n=143) \\
142.38\end{array}$ \\
\hline & $\begin{array}{c}H=32.543 \\
P=0.000 \\
n^{2}{ }_{H}=0.25\end{array}$ & $\begin{array}{c}H=28.360 \\
P=0.000 \\
n^{2}{ }_{H}=0.13\end{array}$ \\
\hline
\end{tabular}

Nota: $\mathrm{n}=$ tamaño muestral; $\mathrm{H}=\mathrm{H}$ de Kruskal Wallis; $\mathrm{p}=\mathrm{p}$ valor. $\mathrm{n} 2 \mathrm{H}=$ eta cuadrada (tamaño del efecto). 
Y en relación al puesto de trabajo, también se hallaron diferencias importantes, siendo los colaboradores que realizan actividades en las vías públicas, seguido de los colaboradores de parques y jardines quienes se encuentran más expuestos y afectados que los colaboradores de servicios generales (pruebas post hoc), encontrando que el nivel de exposición al riesgo aumenta mientras se mantiene mayor contacto con los desechos y residuos que las personas arrojan a las calles y/o contenedores, donde se concentra una gran cantidad de desperdicios y ante la ausencia de normas de higiene, este personal incrementa más aun su nivel de exposición (tamaño del efecto pequeño).

\section{V.CONCLUSIONES}

El impacto del Covid-19 en el mundo ha afectado todos los sectores económicos, sociales, académicos y laborales. Sin embargo el sector laboral es uno de los más difíciles porque las personas se ven en la obligación de continuar en sus funciones pese a los altos riesgos de contagio. La pérdida de empleos a nivel mundial ha sido significativa, lo que conduce a que las condiciones laborales se vean afectadas para los que aún mantienen un trabajo.

El personal de limpieza pública en Arequipa se encuentra expuesto a riesgos laborales por el tipo de trabajo que desarrollan en las vías públicas, al mantener contacto con los desechos o residuos sólidos sin los debidos equipos de protección, existe una alta probabilidad de contraer o contagiarse con el virus Covid-19 y otras enfermedades, un alto porcentaje de estos trabajadores se ha contagiado o afectado por alguna dolencia producto de las condiciones laborales.

Por las condiciones de salud que ha ocasionado la pandemia, resulta inevitable que todas aquellas personas que están obligadas a cumplir largos horarios de trabajo en las calles y en contacto con residuos sólidos, estén estresadas o nerviosas por los contagios, aumentando significativamente la ansiedad y la depresión.

Así mismo, otros riesgos laborales importantes de señalar al que están expuestos los colaboradores son los físicos y ergonómicos, sin dudar su nivel de exposición al medio ambiente, contaminación radiación solar y ruidos, son factores de gran relevancia y que han afectado a un número importante de trabajadores. Otros aspectos importantes son los movimientos que realizan al barrer las vías y espacios públicos, las cargas forzadas que tienen que realizar, que también han generado varias enfermedades ocupacionales como las musculo esqueléticas, digestivas, dermatológicas y respiratorias.

Resulta indispensable que las organizaciones realicen mayores capacitaciones en cuanto a los riesgos laborales actuales, que se han incrementado por la situación de pandemia. Este tipo de formación podría mejorar los actos inseguros y mejorar el correcto uso de equipos de protección. Así mismo las instituciones están obligadas a fortalecer el uso de los recursos de protección, promover políticas de seguridad y contribuir con la capacitación para el uso eficiente de los equipos y evitar la exposición al riesgo.

Los resultados de este trabajo son un precedente para el estudio de la salud laboral, y permite además establecer algunos criterios relevantes para las mejores de las condiciones de trabajo en los colaboradores de la limpieza pública.

Finalmente, el estudio del impacto del Covid-19 en la humanidad, es cada día revelador, no existen estándares que puedan definir la magnitud del alcance. Sin embargo las medidas de seguridad elemental y básica incluyen evitar el exceso de exposición en las calles, y esto conduce a sugerir que las instituciones de limpieza deberían reestablecer las condiciones laborales para reducir las situaciones de salud que enfrentan los trabajadores.

\section{REFERENCIAS}

[1]M. T. Del Campo, «Covid-19 y su impacto en la salud y el trabajo,» Revista de la Asociación Española de Especialistas en Medicina del Trabajo, vol. 29, n 3, p. $183,2020$.

[2]O. Mamani, E. E. Apaza, R. F. Carranza, J. F. Rodriguez y C. R. Mejía, «Inseguridad laboral en el empleo percibida ante el impacto del COVID-19: validación de un instrumento en trabajadores peruanos (LABOR-PE-COVID-19),» Revista de la Asociación Española de Especialistas en Medicina del Trabajo, vol. 29, no 3, pp. 184-193, 2020.

[3]M. Morales, «CONVOCA,» 8 julio 2020. [En línea]. Available: https://convoca.pe/agenda-propia/ covid-19-trabajadores-de-limpieza-publica-el-gremio-que-nunca-pudo-quedarse-en-casa. [Último acceso: 20 Enero 2021].

[4]Ministerio de Salud Pública, «Ministerio de Salud Pública,» 2019. [En línea]. Available: https://www.gob. pe/mtpe. [Último acceso: 12 enero 2021].

[5]A. M. Sánchez, F. J. Sánchez y D. Ruiz, «Riesgos laborales en las empresas de residuos sólidos en Andalucía: una perspectiva de género,» Salud Pública, vol. 26, no 3, pp. 798-810, 2017.

[6]R. Hernández, C. Fernández y P. Baptista, Metodología de la Investigación, México D.F.: McGraw Hill, 2014.

[7]A. Camacho y D. R. Mayorga, «Riesgos laborales psicosociales, perspectiva organizacional, jurídica y so- 
cial,» Prolegómenos - Derechos y Valores, vol. 20, $\mathrm{n}^{\mathrm{o}}$ 40, pp. 159-172, 2017.

[8]R. Jiménez y J. R. Pavés, «Enfermedades y riesgos laborales en trabajadores de servicios de urgencia: revisión de la literatura y acercamiento a Chile,» Medwave, vol. $15, \mathrm{n}^{\circ}$ 7, pp. 1-9, 2015.

[9]J. M. Calizaya, F. M. Zapata y J. L. Evangelista, «Riesgos psicosociales en el trabajo en colaboradores de una empresa minera de Arequipa,» Véritas, vol. 21, $\mathrm{n}^{\mathrm{o}} 2$, pp. 25-30, 2020.

[10]C. E. Venegas y J. E. Cochachin, «Nivel de conocimiento sobre riesgos ergonómicos en relación a síntomas de trastornos músculo esqueléticos en personal sanitario,» Medicina del Trabajo, vol. 28, n 2, pp. 126135, 2019.

[11]M. Sánchez, G. B. Pérez, G. Gonzáles y I. Peón, «Enfermedades actuales asociadas a los factores de riesgo laborales de la industria de la construcción en México,» Medicina y Seguridad del Trabajo, vol. 63, $\mathrm{n}^{\circ}$ 246, pp. 28-39, 2017.

[12]J. Boada, L. Robert, C. Gil y A. Vigil, «Desarrollo, consistencia interna, Habilidad y validez de una escala de riesgos laborales en lengua española,» Anales de Psicología, vol. 29, nº 1, pp. 217-224, 2013.

[13]D. Frías, Análisis de la consistencia interna de las puntuaciones de un instrumento de medida, Valencia: Universidad de Valencia, 2020.

[14]B. Yazici y S. Yolacan, «Una comparación de varias pruebas de normalidad,» Journal of Statistical Computation and Simulation, vol. 77, $\mathrm{n}^{\circ}$ 2, pp. 175-183, 2007. [15]J. L. Ventura, «Tamaño del efecto para la U de Mann Withney: aportes añ articulo de Valdivia-Peralta et al.,» Neuro Psiquiatría, vol. 54, no 4, pp. 353-354, 2016.

[16]S. Dominguez, «Maginitud del efecto, una guía rápida,» Educación Médica, vol. 19, nº 4, pp. 251-254, 2018.

\section{RESUMEN CURRICULAR}

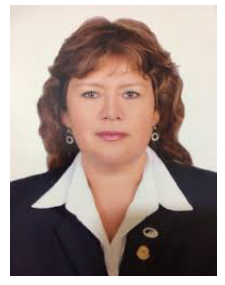

Gloria Isabel Monzón Alvarez. Doctora en Administración (DBA), Magister en Estrategias de Desarrollo y Políticas Sociales, Licenciada en Trabajo Social, integrante de la Unidad de Investigación de la Facultad de Ciencias Histórico-Sociales. Experta en ejecución de Programas de Bienestar Social, Promoción de la Salud y Políticas del Sistema Integral de Salud Pública. Docente en la Escuela Profesional de Trabajo Social de la Universidad Nacional de San Agustín de Arequipa

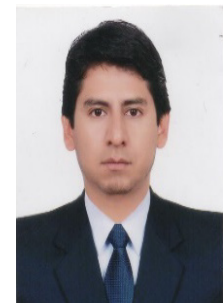

Miguel Ángel Pacheco Quico. Licenciado en Trabajo Social, Docente de la Escuela Profesional de Trabajo Social. Gerente de Desarrollo Social y Económico de Municipalidades Distritales de Arequipa, Planificador Social y Miembro activo del Instituto de Investigaciones Sociales de la Universidad Nacional de San Agustín de Arequipa

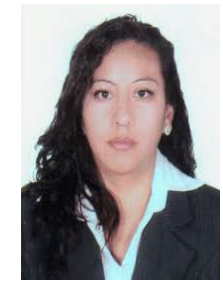

Ana Rosario Miaury Vilca. Magister en Gerencia Social de la Escuela de Posgrado de la Pontificia Universidad Católica del Perú, con estudios en el Doctorado en Gobernabilidad y Gestión Pública Estratégica; Diplomada en Programa de Gobernabilidad y Gerencia Política The George Washington Univesity The Graduate School Of Political Management y la Corporación Andina de Fomento. Docente del Programa de Sociología de la Universidad Nacional de San Agustín de Arequipa

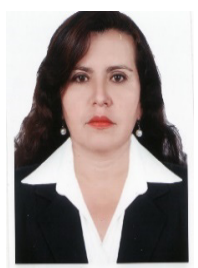

Hilda Lizbeth Pinto Pomareda. Maestra en Ciencias, con mención en Gerencia Social y de Recursos Humanos, con experiencia en el puesto de Gerente Jefe de Departamento y de Área en la Gestión Pública. Docente Asociado en el programa de estudios de Trabajo Social, delegada de los docentes auxiliares ante la Asamblea Universitaria, Docente investigador UNSA- INVESTIGA

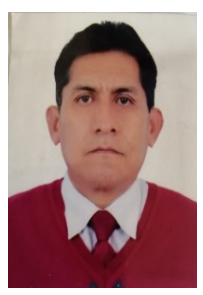

Santiago Felipe Torres Aza. Magister en Gestión de la función docente. Licenciado en Ciencias de la Educación de Universidad Católica Santa María. Licenciado en Ciencias de la Comunicación Social y Diplomado en Didáctica de la Comunicación. Docente actual de Educación Básica Regular. Docente actual del área de Razonamiento Verbal en el Centro Preuniversitario de la Universidad Nacional San Agustín de Arequipa 